\title{
Betatron Radiation of an Off-axis Injected Electron in a Laser Wakefield Accelerator
}

\author{
Seok Won Hwang and Hae June Lee* \\ Department of Electrical Engineering, Pusan National University, Busan 609-735, Korea
}

(Received January 2, 2009 : revised February 16, 2009 : accepted February 17, 2009)

\begin{abstract}
The electrons injected into a laser wakefield undergo betatron oscillation and give rise to the emission of intense X-ray radiation. To investigate the generation conditions of the X-rays, the relativistic motion of an electron injected in an off-axis position has been simulated with wakefield profiles which are pre-calculated with a two-dimensional particle-in-cell code. The wakefield with a plasma density of $1.78 \times 10^{18} \mathrm{~cm}^{-3}$ is generated by the laser with an intensity of $1.37 \times 10^{18} \mathrm{~W} / \mathrm{cm}^{2}$ and a pulse width of $30 \mathrm{fs}$. From the calculation of the single particle motion, the characteristics of the betatron radiation are investigated in the time domain. As the transverse injection position increases, the power and the duration time of the radiation increase, but the width of each pulse decreases.
\end{abstract}

Keywords: Laser wakefield, Betatron radiation, X-ray generation, Electron acceleration

OCIS codes : (350.5400) Plasmas; (340.7480) X-rays; (320.2250) Femtosecond phenomena

\section{INTRODUCTION}

After the invention of ultra-high intensity lasers, the particle acceleration techniques using laser wakefields have been rapidly developed [1]. The laser-induced particle accelerators can have an acceleration gradient of 10-100 $\mathrm{GV} / \mathrm{m}$, which is higher by three orders of magnitude than that of conventional linear accelerators [2]. In addition to the laser-induced accelerator, the laser wakefields can play the same role as wigglers in free electron lasers [3]. An electron in the wakefields is influenced not only by the accelerating force but also by a transverse focusing force. The focusing force is at the same order of magnitude as the accelerating force. Off-axis injected electrons are accelerated by the wakefield and also undergo betatron oscillation. The oscillation gives rise to the emission of intense femtosecond X-ray radiations over a few $\mathrm{keV}$ ranges which have been observed by simulations and experiments [4-7]. Recent experiment shows that a petawatt-laser-generated plasma cavity produces $50 \mathrm{keV}$ X-ray beams [8]. The electron trajectories can be verified from the images of betatron radiations of the electrons in the wakefields [9]. The femtosecond X-ray over a large spectral range can be used for the in-

*Corresponding author: haejune@pusan.ac.kr vestigation of ultrafast transient structures using X-ray diffraction and X-ray absorption techniques [10].

In order to investigate the X-ray generation and to find an optimal condition for strong radiation, it is necessary to understand the electron motion in the wakefield. In this paper, we present the analysis of single electron motion in a laser wakefield accelerator using a two-dimensional particle-in-cell code, assuming the wakefields are not changed for the electron travel period. Sec. II demonstrates the numerical method and conditions used to analyze single electron motion. Sec. III demonstrates the simulation results for the variation of injection positions. The conclusion and discussion are presented in Sec. IV.

\section{SIMULATION CONDITIONS}

For the generation of laser wakefields, we used a two -dimensional electromagnetic particle-in-cell code (XOOPIC) [11]. The simulation was conducted in $x-y$ rectangular coordinates. The simulation parameters are as follows. The electron plasma density is $1.78 \times 10^{18} \mathrm{~cm}^{-3}$ which corresponds to a wakefield wavelength of $\lambda_{p}=$ $25 \mu \mathrm{m}$ and an electron plasma frequency of $\omega_{p}=\left(n_{e} e^{2} /\right.$ $\left.m_{e} \varepsilon_{0}\right)^{1 / 2}=7.5 \times 10^{13} \mathrm{rad} / \mathrm{s}$. The simulation domain is 96 
$\mu \mathrm{m}$ in the $\mathrm{x}$ direction, which is three times the $32 \mu \mathrm{m}$ in the $\mathrm{y}$ direction. This is because the transverse radius of laser wakefields, which is determined by the $4 \mu \mathrm{m}$ size of the waist of the incident laser pulse, is smaller than the size of the wakefield wavelength $\lambda_{p}=25 \mu \mathrm{m}$. The grid size was chosen to be $\Delta x=\Delta y=\lambda / 16$, where $\lambda$ is the laser pulse wavelength $1 \mu \mathrm{m}$. The incident laser pulse has a Gaussian distribution with full-width at half-maximum (FWHM) of 30-fs. The normalized vector potential of the laser pulse $\boldsymbol{a}$ is defined as

$$
\boldsymbol{a}=\boldsymbol{e} / m_{e} c^{2}
$$

The peak amplitude of the normalized vector potential $a_{0}$ is related to the peak intensity $I$ by $a_{0}=8.5 \times 10^{-10}$ $\sqrt{\lambda^{2}[\mu \mathrm{m}] /\left[\mathrm{W} / \mathrm{cm}^{2}\right]}$. The intensity of the laser pulse is $1.37 \times 10^{18} \mathrm{~W} / \mathrm{cm}^{2}$ which corresponds to $a_{0}=1$.

Figure 1 shows three dominant components of the wakefield; (a) longitudinal electric field $E_{x}$, (b) transverse electric field $E_{y}$ and (c) transverse magnetic field $B_{z}$. In cylindrical coordinates, the components correspond to (a) axial electric field, (b) radial electric field and (c) azimuth magnetic field, respectively. The longitudinal field $E_{x}$ is not exactly a sinusoidal wave because of a nonlinear effect. With the increase of $a_{0}$, the nonlinear effect also increases [12]. The minimum magnitude of $E_{x}$ is -34 $\mathrm{GV} / \mathrm{m}$, which is located at $\mathrm{x}=40 \mu \mathrm{m}$ in Fig. 1(a). The profile of the transverse electric field changes along the off-axis and generates focusing and diffracting regions in turn. The maximum magnitude of $E_{y}$ in the accelerating region is $30 \mathrm{GV} / \mathrm{m}$, which is located at a transverse distance of $3 \mu \mathrm{m}$ from the axis. The magnitude of the transverse electric field shows approximately linear increases with the distance near the axis in Fig. 1(b). The profile of the azimuth magnetic field also changes along the off-axis and generates distributions similar to the transverse electric field. However, the sign of the magnetic field is the reverse of the transverse electric field. The maximum magnitude of $B_{z}$ in the focusing region is 15 Tesla at a distance $3 \mu \mathrm{m}$ from the axis. The magnitude of the magnetic field $B_{z}$ also shows linear increases near the axis as the transverse electric field does. For the relativistic electron, the ratio of the maximum magnitude of the Lorentz force from $B_{z}$ fields to that of the $E_{y}$ fields is about $15 \%$ in the case of the given wakefield, and therefore the electric field effect is dominant for the electron motion. However, magnetic fields affect the increase of the transverse force, and thus induce the increase of the oscillation frequency. We will mention the effects of magnetic fields in Sec. III.

The radial electric field and the azimuth magnetic field in the wakefields are related to the axial electric field by the Panofsky-Wenzel theorem, $\partial E_{z} / \partial r=\partial\left(E_{r}-B_{\theta}\right) / / \partial \xi$, where $\xi=z-v_{p} t$ and $v_{p}$ is the wake-field velocity [2]. In this study, the longitudinal

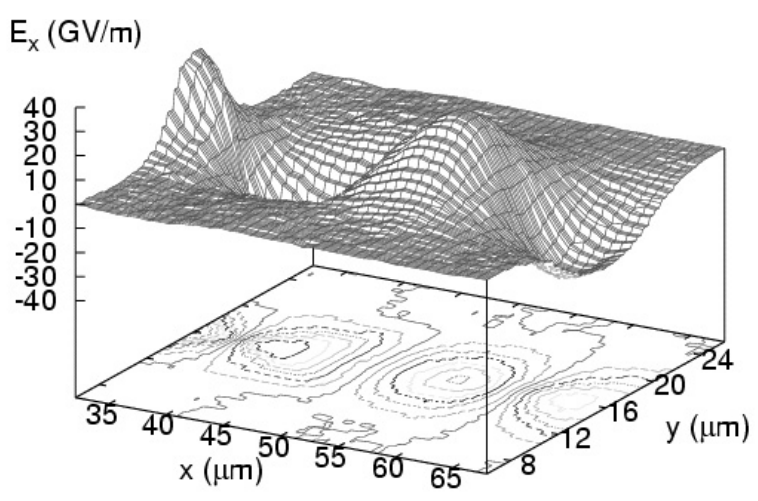

(a)

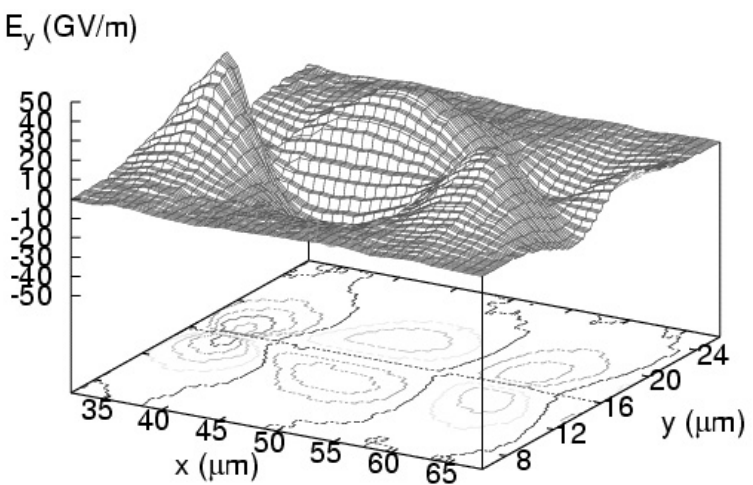

(b)

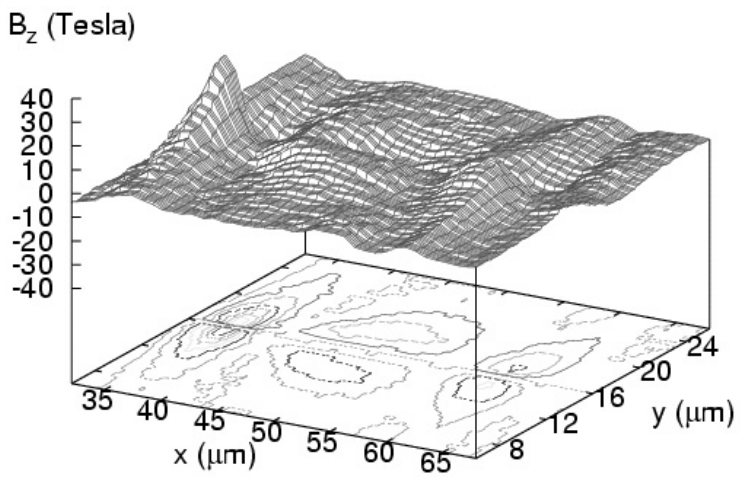

(c)

FIG. 1. The wakefields for the plasma electron density $\mathrm{n}_{\mathrm{e}}=1.78 \times 10^{18} \mathrm{~cm}^{-3}$, which corresponds to the wakefields length $\lambda_{\mathrm{p}}=25 \mu \mathrm{m}$. The wake field has three dominant fields; (a) longitudinal electric fields $\mathrm{E}_{\mathrm{x}}$, (b) transverse electric fields $E_{y}(c)$ azimuth magnetic fields $B_{z}$.

direction is designated with a coordinate $\mathrm{x}$ instead of $\mathrm{z}$, and thus $\xi=x-v_{p}$. The phase velocity of the wakefield is the same as the group velocity of the laser pulse and is given by

$$
v_{p}=c\left(1-\omega_{p}^{2} / \omega^{2}\right)^{1 / 2}
$$

If the wakefields do not change much during the in- 


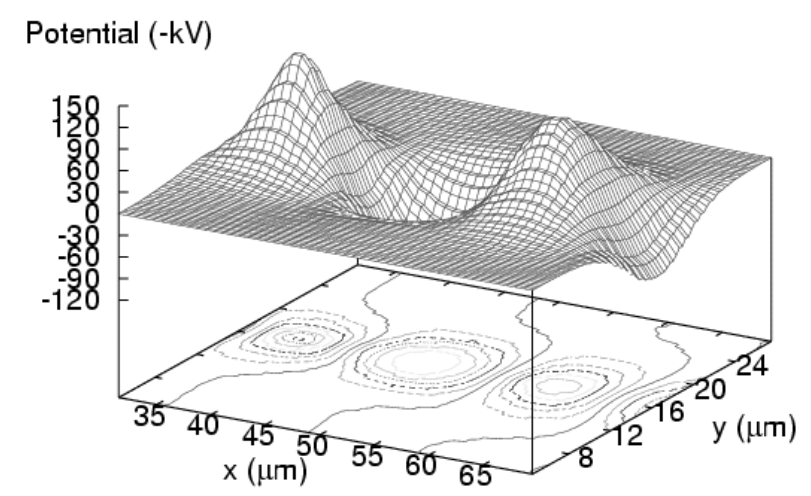

FIG. 2. The profile of the electric potential is calculated from the assumption of the static electric fields.

teraction time, the wakefields can be counted as stationary fields in a moving frame.

Electric field is expressed with an electric scalar potential and a magnetic vector potential as

$$
\boldsymbol{E}=-\nabla V-\frac{\partial \boldsymbol{A}}{\partial t}
$$

If the effect of the electric field is dominant over that of the magnetic field in Eq. (3), the second term in the right-hand-side can be neglected. Figure 2 shows the inverse electric scalar potential profile of the wakefield assuming that the second term of the right-hand side in Eq. (3) is zero. The electric scalar potential can be derived from Poisson's equation,

$$
\nabla^{2} V=-\rho / \varepsilon_{0}
$$

where $\rho$ is the electron charge density and $\varepsilon_{0}$ is the permittivity in vacuum. The electric scalar potential is calculated with a finite difference method. The Neumann boundary condition $\partial E_{x} / \partial n=0$ was adopted at the left and the right boundary. The Dirichlet boundary condition $\mathrm{V}=0$ was adopted at the top and the bottom boundary. The peak-to-peak voltage difference in the axial direction is about $250 \mathrm{kV}$. The cross sectional profile of the potential in the transverse direction at the potential minimum position is a Gaussian distribution with a FWHM of $6.3 \mu \mathrm{m}$, which is approximately 1.5 times larger than the spot size of the laser pulse. At $\xi=0.22 \lambda_{\mathrm{p}}$, The FWHM of cross sectional potential profiles is lower than $2 \mu \mathrm{m}$. This small width restricts the transverse injection position and initial transverse velocity of an electron. The length of the considered region for satisfying acceleration and oscillation simultaneously is one-fourth of the length of the wakefield. If the initial transverse kinetic energy of the electron is not higher than the potential energy, the electron is trapped in the potential well.

In the following section, a single electron motion is described in $\xi$ phase space using static electric wake- fields obtained with XOOPIC code, assuming the wakefields propagate with the velocity of $v_{p}$ in the laser propagation direction. However, for the sake of simple interpretation, the force from magnetic fields is neglected.

\section{SIMULATION RESULT}

The total energy of a single electron is expressed by $E=\gamma m_{e} c^{2}$, where the relativistic factor $\gamma$ is defined as $\gamma=\sqrt{1+u^{2} / c^{2}}$ and the effective velocity $u$ is the product of $\gamma$ and electron velocity $v_{e}$. Figure 3 shows the evolution of the electron velocity for 10 picoseconds (which corresponds to $3 \mathrm{~mm}$ ) with the variation of the transverse injection positions. The wakefield velocity for the electron plasma density $n_{e}=1.7810^{18} \mathrm{~cm}^{-3}$ corresponds to that of an electron with energy $12.77 \mathrm{MeV}$, so the initial energy of the electron was chosen to be 12.77 $\mathrm{MeV}$. In this paper, $\delta_{x}$ is defined as the longitudinal distance between the maximum position of the wake -field potential ( $y=16$ and $x=36 \mu \mathrm{m}$ in Fig. 2 ) and the injection position, and $\delta_{y}$ as the transverse distance between the axis and the injection position. Constant $\delta_{x}$ of $0.26 \lambda_{p}$ is used in Fig. 3.

When $\delta_{y}=0$, the electron does not oscillate because no transverse forces exist on the axis. However, when $\delta_{y}$ has a finite value, the electron experiences a betatron oscillation. Due to the betatron oscillation, the longitudinal velocity of the electron $v_{x}$ is not always higher than the wake-field velocity during the electron travel time except for the case of $\delta_{y}=0$. The longitudinal velocity $v_{x}$ does not increase monotonically but increases with oscillation though the sign of the longitudinal force does not change for the electron travel time. This can be explained as a relativistic effect. The components of relativistic velocity $u_{x}, u_{y}$ and $u_{z}$ are coupled with one another by the $\gamma$ factor, and thus one velocity component affects the other components. The longitudinal relativistic velocity $u_{x}$ and $\gamma$ increase monotonically due to the accelerating field, but the ratio of the $u_{x}$ to $\gamma$ increases with oscillation because transverse relativistic velocity $u_{y}$ oscillates. The frequency of $u_{y}$ is determined by the betatron frequency [5] defined as

$$
\omega_{b}=\omega_{p} / \sqrt{2 \gamma}
$$

The oscillation frequency of $u_{x}$ becomes twice larger than that of the betatron oscillation because $u_{x}$ has its minimum value in one period when $u_{y}$ is 0 . This longitudinal velocity oscillation induces the backward slipping motion in the $\xi$ phase space.

In Fig. 3(b), as the transverse injection position becomes more distant from the axis, the amplitude of $u_{y}$ becomes higher because transverse electric field 


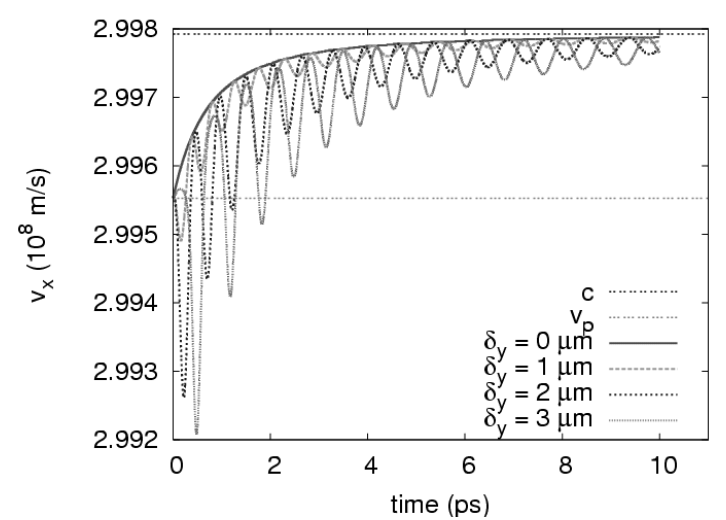

(a)

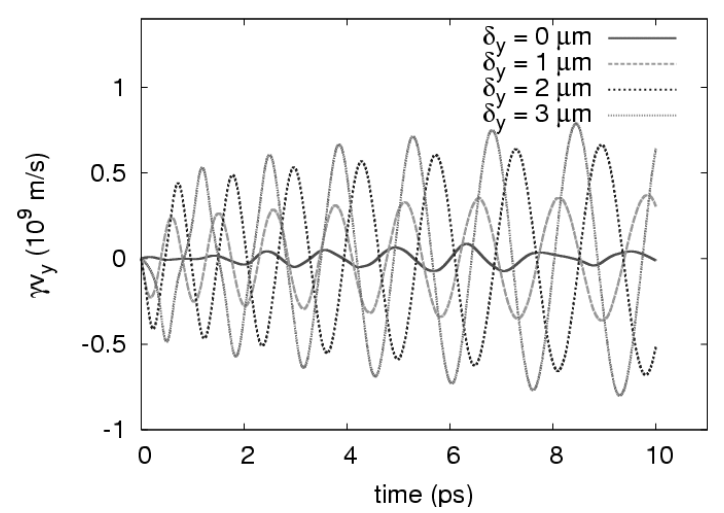

(b)

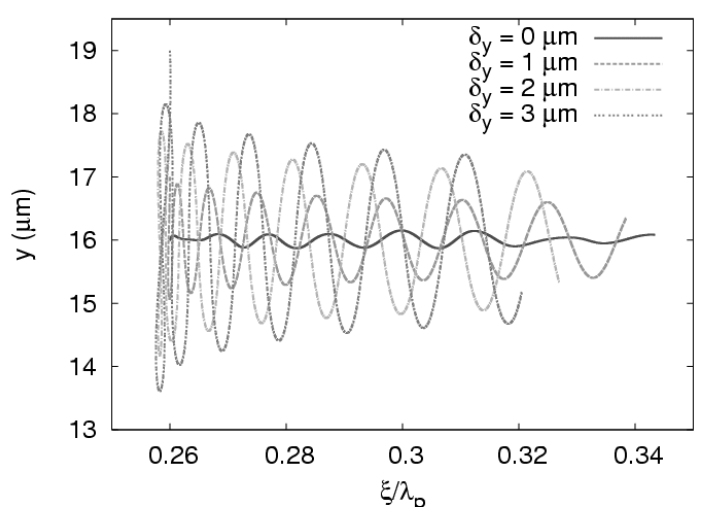

(c)

FIG. 3. The time evolutions of (a) longitudinal velocity and (b) the relativistic transverse velocity of the electron are shown with the variation of the transverse injection position. (c) The electron motion is shown in $\xi$-y phase space. The longitudinal injection position is fixed to be $\delta_{\mathrm{x}}=0.26 \lambda_{\mathrm{p}}$.

increases with radial distance. $u_{x}$ increases monotonically due to the accelerating force. On the other hand, the amplitude of $u_{y}$ does not increase as rapidly as $u_{x}$, and the oscillation amplitude of $v_{x}$ becomes smaller with time evolution. The oscillation frequency of $u_{y}$ decreases by Eq. 5 due to the increase of the electron energy with time evolution. When the

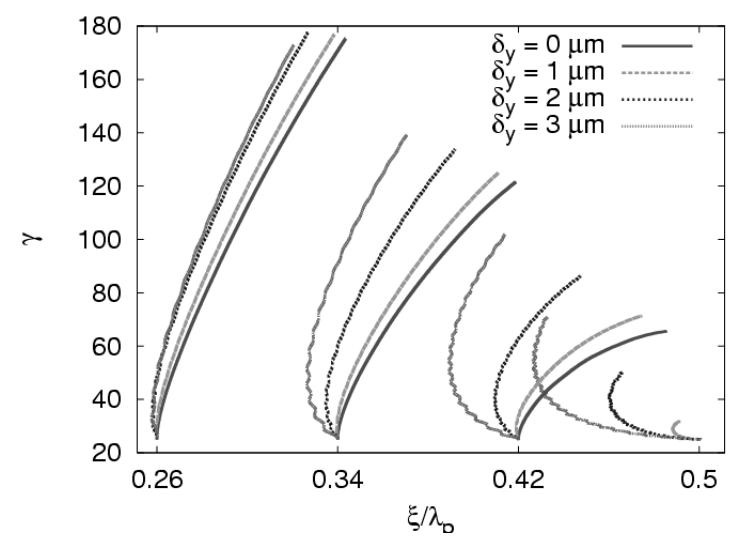

FIG. 4. The electron with initial energy $12.77 \mathrm{MeV}$ is injected at $\delta_{\mathrm{x}}=0.28,0.34,0.42$ and $0.5 \lambda_{\mathrm{p}}$ respectively in the $\xi$ phase space. The transverse injection position $\delta_{y}$ is $0,1,2,3 \mu \mathrm{m}$ respectively. The time evolution of $\gamma$ in the $\xi$ phase space is shown for a travel distance of $3 \mathrm{~mm}$.

transverse distance is same, the transverse force increases as the electron approaches the potential minimum position in Fig. 2. Therefore, the transverse amplitude of the electron decreases in Fig. 3(c). When $\delta_{y}=0$, it is expected to have no oscillation in uy, but a small oscillation is shown in Fig. 3(b) because of defectiveness of axial symmetry.

Figure 4 shows the evolution of $\gamma$ in the $\xi$ phase space with the variation of longitudinal and transverse injection positions for $3 \mathrm{~mm}$ travel distance in a laboratory frame. As the injection position $\delta_{x}$ becomes more distant from the potential minimum position (at $\xi=0.5 \lambda_{p}$ ), the slope of $\gamma$ becomes more steep because the electric field is larger at $\xi=0.25 \lambda_{p}$ than that at $\xi=0.5 \lambda_{p}$. Therefore, the electron initially located at high electric field obtains more energy for the same travel length. When $\delta_{x}=0.26$ $\lambda_{p}$, there is no significant difference in $\gamma$ with the variation of the transverse injection positions, $\delta_{y}$. However, when $\delta_{x}$ is larger than $0.34 \lambda_{p}$, the electron injected in off-axis position significantly slips backward because the transverse electric field increases sharply with the increase of transverse distance and induces the decrease of $v_{x}$.

The electron undergoing betatron oscillation emits radiation at the peak of its transverse position. Figure 5 shows the radiation emitted from the electron in the propagation direction of the laser. If the radiation detector is far away from the radiation source, the detector receives betatron radiation at the time [13] of

$$
t^{\prime}=t-\frac{\hat{\boldsymbol{n}} \cdot \boldsymbol{r}}{c}
$$

If the detector is in the direction of the electron propagation and the electron velocity $v_{e}$ is assumed to be $c$, the total radiation time is determined by 


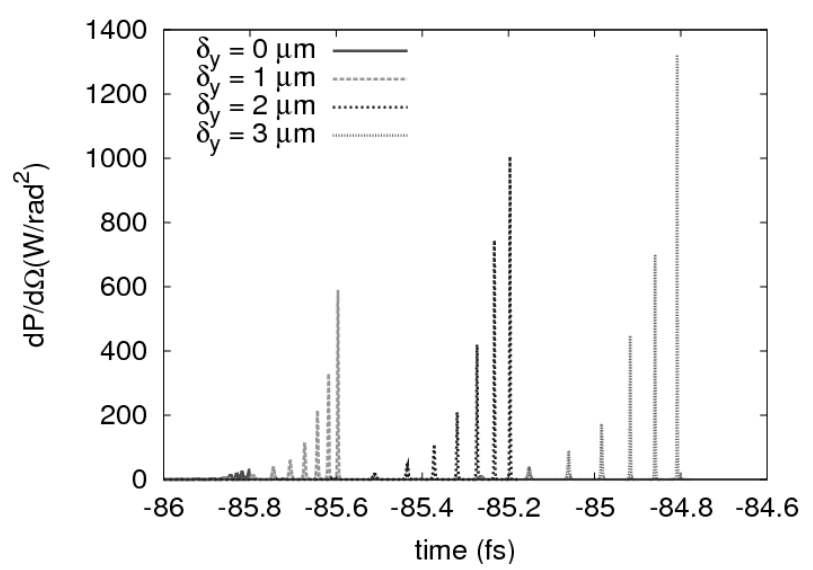

(a)

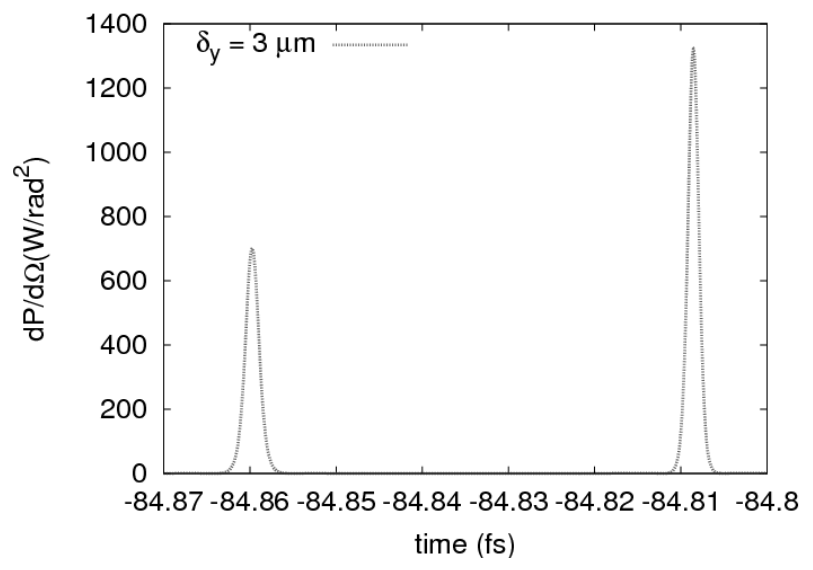

(b)

FIG. 5. (a) The time evolutions of radiation power per solid angle are calculated for different injection positions. (b) The magnified intensity is shown for the case of $\delta_{y}$ $=19 \mu \mathrm{m}$. The radiation is detected in the direction of laser propagation. The total radiation time, the distance between pulses, and the radiation power increase with the transverse injection position.

$$
\Delta t^{\prime} \approx \frac{\Delta t}{2 \gamma^{2}}
$$

where $\Delta t$ is the electron travel time. Therefore, the total radiation time decreases with the increases of electron energy. In Fig. 4, the square of $\gamma$ is $10^{3} \sim 10^{4}$ with the variation of the injection position, and the total radiation time becomes about $1 \sim 10$ fs according to Eq. (7). If the electron energy is maintained approximately constant, the total radiation time is affected by the transverse motion. Because the transverse oscillation makes the longitudinal velocity of the electron $v_{x}$ decrease, the effect of time contraction decreases. For example, when $\delta_{x}=0.26 \lambda_{p}$, the electron injected at $\delta_{y}=0 \mu \mathrm{m}$ propagates longer by $1.5 \mu \mathrm{m}$ than that injected at $\delta_{y}=3$ $\mu \mathrm{m}$, and thus the total duration of the radiation from the latter becomes longer by 5 fs than that from the former.

Each pulse corresponds to a single betatron radiation. The period between pulses is determined by the fundamental frequency as the Doppler shifted betatron frequency [9]

$$
\omega_{f}=\omega_{b}\left(2 \gamma^{2}\right)=(2 / \sqrt{2}) \omega_{p} \gamma^{3 / 2}
$$

As the electron energy increases, the period between pulses becomes smaller. The time distance between pulses is also affected by transverse oscillation as stated above, because for one oscillation period, the electron with the high amplitude of oscillation propagates less distance than that with less amplitude of oscillation.

The width of each pulse is proportional to the inverse of critical frequency [5] defined as

$$
\omega_{c}=(3 / 2 r) \gamma^{3} c=(3 / 2) \gamma^{2}\left|F_{\perp}\right| /\left(m_{e} c\right)
$$

where $r$ is the bending radius of oscillation and $F_{\perp}$ is a transverse force. The maximum transverse force by which the electron is affected does not vary with electron propagation as stated above, but is determined at the time when the electron is injected. It increases with the transverse injection position. Therefore the width of each pulse decreases as $1 / \gamma^{2}$ with the electron propagation. In the case of the simulation parameters considered, $\delta_{x}$ $=0.22 \lambda_{p}$ and $\delta_{y}=2 \mu \mathrm{m}, \gamma$ increases from 25 to 360 at the detuning point, and the maximum of the transverse electric field is $20.0 \mathrm{GeV} / \mathrm{m}$. Then, the critical radiation frequency is expected from $1.1 \times 10^{16}$ to $2.28 \times 10^{18} \mathrm{~s}^{-1}$ which corresponds to the critical energy of $7.2 \mathrm{eV}$ to $1.5 \mathrm{keV}$. Therefore, the X-ray with energy more than $\mathrm{keV}$ is emitted mainly at the end of electron acceleration.

Because the electron propagates with the longitudinal velocity close to the speed of light, the magnetic field contributes to the transverse Lorentz force and thus to the characteristics of the radiation. The total transverse force, which is the sum of forces derived from electric and magnetic fields in the Lorentz equation, increases at most by $15 \%$ more than that for the case considered only with electric field. From the increase of the force, the amplitude of $u_{y}$ and the frequency of betatron oscillation increase. The former results in an effect similar to $\delta_{y}$ increase. The effect of time contraction decreases and the total duration and the critical frequency of the radiation increases. The increase of the frequency contributes a little to the increase of fundamental frequency.

\section{CONCLUSION \& DISCUSSION}

We have discussed the off-axis electron motion under 
the static electric wakefields and the characteristic of the radiation emitted from the betatron oscillation in the time domain. The off-axis injected electron oscillates transversely with a betatron frequency, and the longitudinal velocity oscillates at the double frequency of the betatron frequency. This oscillation induces the decrease of longitudinal velocity of the electron and results in the increase of the total time of the radiation. The oscillation also increases the distance between pulses, however with the increase of amplitude of oscillation, the electron emits the radiation with high power and with high energy. In order to diminish the radiation time and increase total power and energy of radiation, the electron must be accelerated to the high energy.

In practical applications, the radiation is emitted not only from one electron but from many electrons which are captured within the wakefield at widespread locations. Therefore, the coherency issue of the radiation is very important. It was observed from simulation that the coherency of the radiation is not good for multiple electrons. This issue will be explored in further research.

\section{REFERENCES}

1. W. P. Leemans, B. Nagler, A. J. Gonsalves, Cs. Toth, K. Nakamura, C. G. R. Geddes, E. Esarey, C. B. Schroeder, and S. M. Hooker, "GeV electron beams from a centimeter -scale accelerator," Nature Physics 2, 696-699 (2006).

2. E. Esarey and P. Sprangle, "Overview of plasma-based accelerator concepts,” IEEE Trans. Plasma Sci. 24, 2 (1996).

3. S. Wang, et al, "Observation of spontaneous emitted x-ray betatron radiation in beam-plasma interactions," in Proc. 2001 Particle Accelerator Conference (Chicago, USA, 2001), pp. 3999-4001.

4. E. Esarey, B. A. Shadwick, P. Catravas, and W. P.
Leemans, "Synchrotron radiation from electron beams in plasma-focusing channels," Phy. Rev. E 65, 056505 (2002).

5. A. R., K. T. Phuoc, R. Shah, A. Pukhov, E. Lefebvre, V. Malka, S. Kiselev, F. Burgy, J.-P. Rousseau, D. Umstadter, and D. Hulin, "Production of a keV x-ray beam from synchrotron radiation in relativistic laserplasma interaction," Phy. Rev. Lett. 93, 13 (2004).

6. W. P. Leemans, E. Esarey, J. van Tilborg, P. A. Michel, C. B. Schroeder, C. Toth, C. G. R. Geddes, and B. A. Shadwick, "Radiation from laser accelerated electron bunches: coherent terahertz and femtosecond x-rays," IEEE Trans. Plasma Sci. 33, 1 (2005).

7. K. T. Phuoc, F. Burgy, J.-P. Rousseau, V. Malka, and A. Rousse, "Laser based synchrotron radiation," Phy. Plasmas 12, 023101 (2005).

8. S. Kneip, S. R. Nagel, C. Bellei, N. Bourgeois, A. E. Dangor, A. Gopal, R. Heathcote, S. P. D. Mangles, J. R. Marques, A. Maksimchuk, P. M. Nilson, K. Ta Phuoc, S. Reed, M. Tzoufras, F. S. Tsung, L. Willingale, W. B. Mori, A. Rousse, K. Krushelnick, and Z. Najmudin, "Observation of synchrotron radiation from electrons accelerated in a petawatt-laser-generated plasma cavity, Phys. Rev. Lett. 100, 105006 (2008).

9. K. T. Phuoc, S. Corde, R. Shah, F. Albert, R. Fitour, J.-P. Rousseau, F. Burgy, B. Mercier, and A. Rousse, "Imaging electron trajectories in a laser-wakefield cavity using betatron x-ray radiation,” Phy. Rev. Lett. 97, 225002 (2006).

10. A. Rousse, K. Ta Phuoc, R. Shah, R. Fitour, and F. Albert, "Scaling of betatron x-ray radiation," Eur. Phys. J. D 45, 391-398 (2007).

11. J. P. Verboncoeur, A.B. Langdon, and N. T. Gladd, "An object-oriented electromagnetic PIC code," Comp. Phys. Comm. 87, 199-211 (1995).

12. P. Sprangle, E. Esarey, and A. Ting, "Nonlinear theory of intense laser-plasma interactions," Phys. Rev. Lett. 64, 17 (1990).

13. J. D. Jackson, Classical Electrodynamics, $3^{\text {rd }}$ Edition (John Wiley \& Sons, Inc., 1999). 